\title{
Role of MicroRNA in atrial fibrillation: What are the implications for gene therapy?
}

\section{Editorial}

It is well known that the genesis of atrial fibrillation (AF) is multifactorial. The necessity of an atrial myocardial substrate and the presence of triggers are paramount in the development of AF. ${ }^{1-7}$ In addition, efforts have focused on genetics for a better comprehension of the molecular basis of AF, in order to deal properly with this dreadful tachyarrhythmia. Interesting studies dealing with genomewide association differenciated at least 14 distinctive genetic loci interrelated with $\mathrm{AF}{ }^{8}$

Pharmacological therapy is widely utilized for rhythm and rate control of $\mathrm{AF}$, despite the mild efficacy and several colateral effects, including pro-arrhythmic effects. On the other side, nonpharmacological therapy, namely, AF catheter ablation is effective in young patients with paroxysmal AF but not in other clinical presentations of the arrhythmia. ${ }^{9}$ Therefore, it is a necessity to develop new effective treatments that could be beneficial and widely applied in an individual manner in AF patients. This is currently intensively investigated in different scenarios.

The utilization of targeted genetic abnormalities to personalize AF therapy is a compelling approach in current era of efforts to individualize the best therapeutic option for a certain patient. There are certain advantages and disadvantages in the implementation of gene therapy for AF. ${ }^{10-12}$ Some advantages comprise the fact that is tissue specific with fewer side effects. Due to the fact that the AF substrate is not homogeneous, the efficacy of a single genetic alteration may decrease, and it may preclude the simplicity of the procedure.

Concerns about the form of delivery of the genetic material still remain. The therapeutic genetic material may be delivered through viral vectors, or plasmids, or nanoparticles. Viral vectors can incorporate the genetic material into the genome of the tissue that it is been targeted. Although viral vectors may be the most functional, reasonable preoccupation about their safety exists. A non-viral vector consists of a DNA plasmid that harbor the gene of interest, which may contain other coating agents as well to improve the uptake of DNA into cells. ${ }^{10}$ Both adenoviral vectors and adeno-related viral vectors have been utilized in preclinical models and have their advantages and disadvantages. ${ }^{11}$ The adenovirus has the advantage and capability of delivering larger gene sizes. The adeno-related viral vectors may generate longer gene expression and is related to better safety profile. ${ }^{12}$ Many challenges in the development of gene therapy still remain. Effectivity and constancy has proven ambiguous and evasive in AF gene therapy. Hence, there is an increasing necessity of innovative approaches in the therapeutic management in AF patients.

The microRNAs are short, non-coding RNAs with a size of 19-25 nucleotides that has attracted the most attention in recent years since its discovery more than two decades ago. Londin E, et al. ${ }^{12}$ suggested that there are more than 5,000 microRNAs in the human genome. Each individual microRNA controls a family of genes, and individual genes may be controlled by multiple microRNAs. This complicated and finely coordinated system adjusts gene expression in different conditions such as development, stress conditions, and disease.
Volume I4 Issue 3 - 202 I

\author{
Osmar Antonio Centurión, ${ }^{1,2}$ Christian 0 \\ Chávez' \\ 'Department of Cardiovascular Medicine, Clinical Hospital, \\ Paraguay \\ ${ }^{2}$ Department of Health Sciences Investigation, Sanatorio \\ Metropolitano, Paraguay
}

Correspondence: Osmar Antonio Centurión, MD, PhD, FAHA, FACC Professor of Medicine. Asunción National University (UNA). Department of Health Science's Investigation, Sanatorio Metropolitano, Paraguay, Email osmarcenturion@hotmail.com

Received:June 15, 2021 | Published: June 28, 202 I

MicroRNAs are increasingly notable for playing an important role in the pathogenesis of AF. Animal experimental studies have shown promising results in the utilization of gene therapy for AF. In a porcine model of AF, genetic knockdown of caspase-3, an apoptotic enzyme, with an adenovirus-mediated silencing RNA produced atrial conduction delay and development of AF episodes with rapid atrial pacing. ${ }^{13}$ In a study of canine model with a lentivirus containing microRNA-206 gene therapy produced abbreviation of the atrial action potential duration to a greater extent of the one seen with atrial tachy-pacing alone. Therefore, gene therapy with a lentivirus bearing the antagonist anti-microRNA-206 produced prolongation of the atrial refractoriness and decreased $\mathrm{AF}$ inducibility. ${ }^{14}$

MicroRNAs are considered to play an important role in all cardiac remodeling processes and especially in fibrotic responses, including those related with AF. ${ }^{15-19}$ Most fibrosis regulating microRNAs directly bind to pro-fibrotic targets. Their down- regulation liberates these pathways from inhibition, resulting in increase of fibrosis promoting pathways. ${ }^{15}$ Certain entities such as heart failure, pericarditis, and rapid atrial activation are 3 paradigms that promote increasing atrial microRNA-21 expression, development of AF, and can lead to atrial fibrosis. In another study, restoration of atrial microRNA-21 expression back to control levels by left atrial injection of an antimicroRNA that down-regulates microRNA-21 represses fibrosis of the atrial myocardium and precludes AF induction in experimental post-myocardial infarction in rats. ${ }^{16}$ The balance of anti-fibrotic microRNAs and pro-fibrotic microRNAs determines the activation of fibroblasts, the acceleration of fibrotic signaling, the accumulation of extracellular matrix components and fibrotic processes in cardiac remodeling and arrhythmias.

Researches are actively paving the way to modulate microRNA signaling to search for adequate therapeutic management. However, major challenges are set by the multiplicity of gene targets for each individual microRNAs. ${ }^{17}$ The multiple microRNAs altered by any 
one condition, and the involvement of many microRNAs in multiple tissues and conditions, are making specific gene targeting difficult. In the atrial myocardium of heart failure patients there are at least 8 fibrosis-implicated microRNAs that change significantly, raising doubts about the effectiveness of targeting a single microRNA. ${ }^{18,19}$ Therefore, it seems that despite a great distance has been traveled so far in this field of gene therapy, there still remain significant challenges that must be conquered before its clinical utilization will become a reality and be widely open. Nevertheless, the therapeutic management of AF remains in crucial necessity of novel treatment paradigms, and gene therapy potentially offers some distinctive advantages that may be useful in the treatment of AF. Gene therapy has already shed some additional light in the management of $\mathrm{AF}$, and may provide unique opportunities to increase our comprehension of this dreadful arrhythmia and expand our treatment options. However, there is still a long way to go until gene therapy will be broadly implemented in rutine clinical practice to individually treat AF patients.

\section{Acknowledgments}

None.

\section{Conflicts of interest}

The rest of the authors declare do not have conflicts of interest.

\section{Funding}

This manuscript was not supported by any funding.

\section{References}

1. Haïssaguerre M, Jaïs P, Shah DC, et al. Spontaneous initiation of atrial fibrillation by ectopic beats originating in the pulmonary veins. $N$ Engl J Med. 1998;339(10):659-666.

2. Centurión OA, Shimizu A, Isomoto S, et al. Influence of advancing age on fractionated right atrial endocardial electrograms. Am J Cardiol. 2005;96(2):239-242.

3. Centurion OA, Isomoto S, Shimizu A, et al. The effects of aging on atrial endocardial electrograms in patients with paroxysmal atrial fibrillation. Clin Cardiol. 2003;26(9):435-438.

4. Centurión OA, Shimizu A, Isomoto S, et al. Mechanisms for the genesis of paroxysmal atrial fibrillation in the Wolff- Parkinson-White syndrome: Intrinsic atrial muscle vulnerability vs. electrophysiological properties of the accessory pathway. Europace. 2008;10(3):294-302.

5. Centurion OA, Fukatani M, Konoe A, et al. Different distribution of abnormal endocardial electrograms within the right atrium in patients with sick sinus syndrome. Br Heart J. 1992;68(6):596-600.
6. Moe GK, Abildskov JA. Atrial fibrillation as a self-sustaining arrhythmia independent of focal discharge. Am Heart J. 1959;58(1):59-70.

7. Tucker NR, Clauss S, Ellinor PT. Common variation in atrial fibrillation: navigating the path from genetic association to mechanism. Cardiovasc Res. 2016;109(4):493-501.

8. Bhargava M, Di Biase L, Mohanty P, et al. Impact of type of atrial fibrillation and repeat catheter ablation on long-term freedom from atrial fibrillation: results from a multicenter study. Heart Rhythm. 2009;6(10):1403-1412.

9. Donahue JK. Biological therapies for atrial fibrillation: ready for prime time? J Cardiovasc Pharmacol. 2016;67(1):19-25.

10. Kikuchi K, McDonald AD, Sasano T, et al. Targeted modification of atrial electrophysiology by homogeneous transmural atrial gene transfer. Circulation. 2005;111(3):264-270.

11. Igarashi T, Finet JE, Takeuchi A, et al. Connexin gene transfer preserves conduction velocity and prevents atrial fibrillation. Circulation. 2012;125(2):216-225

12. Londin E, Loher P, Telonis AG, et al. Analysis of 13 cell types reveals evidence for the expression of numerous novel primate- and tissuespecific microRNAs. Proc Natl Acad Sci U S A. 2015;112(10):E1106E1115.

13. Trappe K, Thomas D, Bikou O, et al. Suppression of persistent atrial fibrillation by genetic knockdown of caspase 3: a pre-clinical pilot study. Eur Heart J. 2013;34(2):147-157.

14. Zhang Y, Zheng S, Geng Y, et al. MicroRNA profiling of atrial fibrillation in canines: miR-206 modulates intrinsic cardiac autonomic nerve remodeling by regulating SOD1. PLoS One. 2015;10(3):e0122674.

15. Dawson K, Wakili R, Ordög B, et al. MicroRNA 29: a mechanistic contributor and potential biomarker in atrial fibrillation. Circulation. 2013;127(24):1466-1475.

16. Huang Z, Chen XJ, Qian C, et al. Signal transducer and activator of transcription 3/microRNA-21 feedback loop contributes to atrial fibrillation by promoting atrial fibrosis in a rat sterile pericarditis model. Circ Arrhythm Electrophysiol. 2016;9(7):e003396.

17. Cardin S, Guasch E, Luo X, et al. Role for MicroRNA-21 in atrial pro-fibrillatory fibrotic remodeling associated with experimental postinfarction heart failure. Circ Arrhythm Electrophysiol. 2012;5(5):10271035 .

18. Luo X, Yang B, Nattel S. MicroRNAs and atrial fibrillation: mechanisms and translational potential. Nat Rev Cardiol. 2015;12(2):80-90.

19. Chen Y, Wakili R, Xiao J, et al. Detailed characterization of microRNA changes in a canine heart failure model: relationship to arrhythmogenic structural remodeling. J Mol Cell Cardiol. 2014;77:113-124. 